\title{
Postcontrast T1 Mapping for Differential Diagnosis of Recurrence and Radionecrosis after Gamma Knife Radiosurgery for Brain Metastasis
}

\author{
(D). Wang, (D) Y. Zhang, (D) B. Zhao, (D) P. Zhao, (D) M. Ge, (D) M. Gao, (D). Ding, (D) Xu, and (D). Liu \\ O- $=$
}

\begin{abstract}
BACKGROUND AND PURPOSE: The differential diagnosis of radionecrosis and tumor recurrence in brain metastases is challenging. We investigated the diagnostic efficiency of postcontrast $\mathrm{Tl}$ mapping in solving this problem.
\end{abstract}

MATERIALS AND METHODS: Between March 2016 and June 2017, fifty-six patients with brain metastases who underwent contrastenhanced cerebral T1 mapping were recruited for this prospective study. The findings revealed new enhancement after gamma knife radiosurgery. The subjects were assigned to radionecrosis and recurrence groups based on follow-up (median, 11.5 months) and histopathologic results. $T 7$ values of lesions $5\left(T_{5 \mathrm{~min}}\right)$ and $60\left(\mathrm{Tl}_{60 \mathrm{~min}}\right)$ minutes after administration of contrast agent and their difference $\left(T 1_{\text {differ }}\right)$ were compared between the 2 groups with the 2-tailed Mann-Whitney $U$ test. Receiver operating characteristic curves were used to determine the optimum cutoff values for differential diagnosis.

RESULTS: There were significant differences between the 2 groups in $\mathrm{Tl}_{5 \mathrm{~min}}, \mathrm{Tl}_{60 \mathrm{~min}}$, and $\mathrm{Tl}_{\text {differ }}$ values $(P=.012, P=.004$, and $P<.001$, respectively). Relative to $\mathrm{Tl}_{5 \mathrm{~min}}$ and $\mathrm{Tl}_{60 \mathrm{~min}}, \mathrm{Tl}_{\text {differ }}$ exhibited greater sensitivity and specificity $(P<.001$, respectively) in identifying radionecrosis. The optimum $\mathrm{Tl}_{\text {differ }}$ value for differential diagnosis was $71.1 \mathrm{~ms}$ (area under the curve $=0.97 ; 95 \% \mathrm{Cl}, 0.93-1.00$ ), with sensitivity and specificity of $81.5 \%$ and $96.5 \%$, respectively.

CONCLUSIONS: Postcontrast T1 mapping is optimal for the differential diagnosis of radionecrosis and tumor recurrence. Among $\mathrm{Tl}$ parameters, $\mathrm{Tl}_{\mathrm{differ}}$ is the most powerful parameter for differential diagnosis. Advantages in terms of quantitative analysis and high resolution portend the wide use of postcontrast $T 1$ mapping in the future.

ABBREVIATIONS: $\mathrm{AUC}=$ area under the curve; $\mathrm{BM}=$ brain metastases; $\mathrm{GKR}=$ gamma knife radiosurgery; $\mathrm{rCBV}=$ relative cerebral blood volume; $\mathrm{Tl}_{5 \mathrm{~min}}=\mathrm{T}$ values at 5 minutes after contrast administration; $T_{60 \text { min }}=T$ values at 60 minutes after contrast administration; $T_{\text {differ }}=$ the difference between $T_{60 \mathrm{~min}}-T_{5 \mathrm{~min}}$

B rain metastases $(\mathrm{BM})$ are the most common tumors of the central nervous system. The presence of metastatic disease in the CNS portends a poor prognosis and is a leading cause of mor-

Received November 9, 2017; accepted after revision March 3, 2018.

From the School of Medicine (B.W.), and Shandong Medical Imaging Research Institute (Y.Z., B.Z.), Shandong University, Jinan, P.R. China; and Department of Neurosurgery (P.Z., M.Ge, M.Gao, F.D., S.X., Y.L.), Provincial Hospital Affiliated with Shandong University, Jinan, P.R. China.

We gratefully acknowledge the financial support of the National Natural Science Foundation of China (81641176), the Natural Science Foundation of Shandong Province (ZR2014HM069 and ZR20141TM002), the Science and Technology Planning Project of Shandong Province (2015GSF118161, 2017CXGC1209, 2014GSF118046, and 2016GSF201092), The National Key Research and Development Program (2016YFC0106105). and the Taishan Scholars Program (No. tsqn20161070).

Please address correspondence to Yingchao Liu, MD, or Shangchen Xu, MD, Department of Neurosurgery, Provincial Hospital Affiliated with Shandong University, Jingwu Rd 324, Jinan, 250021 P.R. China; e-mail: Yingchao Liu, 13805311573@126.com, and Shangchen Xu, Shangchenxu@sina.com

-- Indicates open access to non-subscribers at www.ajnr.org

三 Indicates article with supplemental on-line tables.

http://dx.doi.org/10.3174/ajnr.A5643 bidity and mortality. ${ }^{1}$ Gamma knife radiosurgery (GKR) has become a popular treatment option for BM because it has good performance in local tumor control and results in improved quality of life and prolonged survival. ${ }^{2,3}$ Despite these benefits, GKR is associated with a risk of deleterious effects on surrounding healthy tissue, which results in the so-called radiation damage. ${ }^{4}$ Radionecrosis results from late-delayed radiation damage, which occurs 6 weeks to months after radiation treatment for BM. ${ }^{5} \mathrm{MR}$ imaging is considered a standard method of follow-up examination after GKR. However, conventional MR imaging findings pose a challenge for distinguishing radionecrosis and tumor recurrence. ${ }^{6}$ Because management strategies for radionecrosis and tumor recurrence are completely different, it is crucial to improve the diagnostic accuracy of MR imaging to ensure further optimal treatment. ${ }^{7}$

Advanced MRI techniques are regarded as powerful tools for improving the diagnostic accuracy of radionecrosis and tumor recurrence. ${ }^{8,9}$ Dynamic susceptibility-weighted contrast-enhanced perfusion MR imaging is the most common method of advanced 
imaging. However, previous studies have found that it was difficult to make a differential diagnosis between radionecrosis and tumor recurrence when the values of relative CBV ( $\mathrm{rCBV}$ ) are in the range of $1.4-2.1^{10,11}$ and the range of uncertainty is even greater in practice, especially when one takes into account multiple institutions and variable analyses. In addition, DSC perfusion MR imaging is highly susceptible to hemosiderin deposition adjacent to vessels or fissures. ${ }^{8,11}$ Dynamic contrast-enhanced perfusion imaging has become increasingly popular for this differential diagnosis. ${ }^{12,13}$ It measures the changes in T1 relaxation associated with the disrupted blood-brain barrier following contrast administration. However, some degree of overlap between the 2 disease entities has been observed in most studies. ${ }^{14}$ Moreover, dynamic contrast-enhanced perfusion also has the disadvantages of low imaging resolution and high sensitivity to susceptibility artifacts in comparison with routine MR images. Findings on the efficiency of apparent diffusion coefficient maps and fractional anisotropy values derived from diffusion tensor imaging are controversial $^{15,16}$ because of their low sensitivity and specificity in assessing the treatment response after GKR. MR spectroscopy has also been used in many studies; however, because of its low spatial resolution, signal-to-noise ratio, and reproducibility, this method is not considered a feasible tool for assessing the treatment response. ${ }^{17,18}$ In addition, none of the abovementioned approaches simultaneously provide high resolution and quantitative assessment. It is therefore necessary to develop a quantitative method for improving the accuracy of the differential diagnosis after GKR.

Late gadolinium enhancement is a useful technique for detection of myocardial scarring. ${ }^{19}$ Zach et $\mathrm{al}^{20}$ reported that delayedcontrast MR imaging for calculating high-resolution treatmentresponse assessment maps could clearly differentiate tumor/ nontumor tissue in patients with brain tumor. They suggested that the delayed enhancement was a result of damaged vessel lumens that are unable to provide efficient clearance of contrast from the tissues, resulting in delayed contrast accumulation. ${ }^{21}$ Recent pathologic findings have also demonstrated the presence of gliosis and scarring in radionecrotic lesions. ${ }^{22,23}$ Tumor recurrence is characterized by neovascularization and cell proliferation. The difference in vascularity and the extravascular, extracellular space in the 2 entities may result in the different contrast enhancement kinetics. Although profound, their work was based on a qualitative method, which may fare poorly in comparisons across patients and institutions. Therefore, we wanted to validate a quantitative method to differentiate radionecrosis from tumor recurrence on the basis of pathologic differences. The concentration of the contrast agent can influence T1 relaxation times of tissues. Fortunately, the T1 mapping - a MR imaging technique that enables quantitative evaluation of T1 relaxation times of tissues - would help reflect the pathologic differences in lesions. In this study, we explored the possibility and efficiency of postcontrast T1 mapping as a treatment response indicator after GKR for $\mathrm{BM}$ in primary clinical applications.

\section{MATERIALS AND METHODS Study Population}

Written informed consent was obtained from all volunteers before commencement of the study and after receiving approval from the ethics committee of the Shandong Provincial Hospital.
All experiments were performed in compliance with the Declaration of Helsinki.

Between March 2016 and June 2017, a total of 78 patients recruited from our hospital were evaluated by postcontrast $\mathrm{T} 1$ mapping. The inclusion criteria were as follows: 1) histopathologic diagnosis of primary cancer; 2) solitary brain metastases that met the criterion of Response Assessment in Neuro-OncologyBrain Metastases on primary MR images; 3) treatment by only GKR; 4) newly enhanced lesions (lost enhancement and gained it back, or with enlarging enhancement) revealed inside the irradiated nidus after injection of contrast agent during follow-up MR imaging examination; and 5) a postirradiation period of $>5$ months. We excluded 22 patients for imaging quality (artifacts, low signal-tonoise ratio) and MR imaging contraindications. Finally, 56 patients (30 women; 26 men; median age, 59 years; age range, $31-80$ years) were included in this study.

\section{MR Imaging}

All patients were imaged in the supine position with a 3T MR imaging machine (Magnetom Skyra; Siemens, Erlangen, Germany) using a transmit-receive quadrature 16-channel head-andneck coil. The imaging protocol was the same for all patients. Dynamic susceptibility-weighted contrast-enhanced perfusion images were acquired after administration of $0.1 \mathrm{mmol}$ per $\mathrm{kg}$ of body weight of Gd-DTPA, followed by a 20-mL saline flush with an injection velocity of $4.0 \mathrm{~mL} / \mathrm{s}$; these images were acquired during 50 scan phases. T1-mapping images were acquired 5 minutes after Gd-DTPA administration for a total scan time of 1 minute. Routine T1WI was performed after the first T1 mapping. Finally, with the same T1-mapping sequence as before, images were acquired 60 minutes after Gd-DTPA administration. A gamma knife rigid head frame, which was matched with the head coil, was fixed on the patient's head. The Brain Dot Engine technique (Siemens) was used for the registration. These measures could guarantee identical slice position and angulation when the patient was repositioned between the 5- and 60-minute time points. Slice positions for all imaging sequences were identical during the entire scan.

\section{T1-Mapping Sequence}

This volumetric multisection quantitative MR imaging pulse sequence, which combined the principles of T1-weighting and MR imaging, was used for a single acquisition. This sequence had 2 flip angles and thus generated 2 self-registered images per section, each with different levels of T1-weighting. These 2 acquired images were processed to generate quantitative MR imaging maps that portray $\mathrm{T} 1$ distribution. The technique was described previously. ${ }^{24}$ To guarantee the accuracy of T1 values, we used actual flip angle imaging, which is a steady-state $3 \mathrm{D}$ echo-spoiled gradient echo $B_{1}$ mapping method based on a dual-repetition time $(T R)$ acquisition $\left(T R_{2}=N \times T R_{1}\right.$, where $N$ is typically an integer on the order of 5) for the bias correction in T1 mapping. ${ }^{25}$ In addition, a water phantom scan was performed for the correction of the magnetic field and radiofrequency field before the MR imaging examination. Last, 2 ROIs, which were completely symmetric, were separately placed on both sides of normal-appearing white matter. If the T1 difference between these ROIs was $<50$ 
Table 1: Clinical characteristics of the study population ${ }^{\mathrm{a}}$

\begin{tabular}{lccc}
\hline \multicolumn{1}{c}{ Variables } & Radionecrosis & Recurrence & Summary \\
\hline Age (IQR) (yr) & $58(52.5-66)$ & $59(53-67)$ & $58.5(53-65.8)$ \\
Sex (M/F) & $13: 16$ & $13: 14$ & $26: 30$ \\
Primary tumor history & & & \\
$\quad$ Lung (No.) (\%) & $18(62.0)$ & $16(59.3)$ & $34(60.7)$ \\
Digestive tract (No.) (\%) & $2(6.9)$ & $4(14.8)$ & $6(10.6)$ \\
Breast (No.) (\%) & $4(13.8)$ & $4(14.8)$ & $8(14.3)$ \\
Kidney (No.) (\%) & $2(6.9)$ & $2(7.4)$ & $4(7.1)$ \\
Skin (No.) (\%) & $3(10.3)$ & $1(3.7)$ & $4(7.1)$ \\
Location & & & \\
Occipital (No.) (\%) & $7(24.1)$ & $4(14.8)$ & $11(19.6)$ \\
Parietal (No.) (\%) & $5(17.2)$ & $7(25.9)$ & $12(21.4)$ \\
Frontal (No.) (\%) & $7(24.1)$ & $5(18.5)$ & $12(21.4)$ \\
Temporal (No.) (\%) & $3(10.3)$ & $2(7.4)$ & $5(18.5)$ \\
Cerebellum (No.) (\%) & $4(13.8)$ & $6(22.2)$ & $10(37.0)$ \\
Brain stem (No.) (\%) & $1(3.4)$ & $1(3.7)$ & $2(7.4)$ \\
Basal ganglia (No.) (\%) & $2(6.9)$ & $2(7.4)$ & $4(14.8)$ \\
MD (median) (IQR) (cm) & $2.2(1.6-2.5)$ & $2.5(1.7-3.4)$ & $2.3(1.6-2.8)$ \\
Dose (median) (IQR) (Gy) & $18(18-21)$ & $18(18-21)$ & $18(18-21)$ \\
KPS (median) (IQR) & $70(70-90)$ & $70(60-90)$ & $70(70-90)$ \\
\hline Not & & &
\end{tabular}

Note:-KPS indicates Karnofsky Performance Status Scale; MD, maximum diameters; IQR, interquartile range.

${ }^{a}$ Data are presented as descriptive statistics (median or count). Numbers in parentheses represent the range of data.

Table 2: Radiographic characteristics of the study population

\begin{tabular}{lccc}
\hline \multicolumn{1}{c}{ Parameters } & Radionecrosis & $\begin{array}{c}\text { Tumor } \\
\text { Recurrence }\end{array}$ & $\boldsymbol{P}$ Value \\
\hline $\mathrm{TI}_{5 \min }$ (median) (IQR) (ms) & $914(824-1055)$ & $817(567-924)$ & .012 \\
$\mathrm{TT}_{60 \text { min }}$ (median) (IQR) (ms) & $798(682-920)$ & $981(774-1160)$ & .004 \\
$\mathrm{TT}_{\text {differ }}$ (median) (IQR) (ms) & $-126(-276$ to -79$)$ & $214(109-269)$ & $<.001$ \\
$\mathrm{rCBV}$ (median) (IQR) & $1.12(0.86-1.35)$ & $2.57(2.15-3.13)$ & $<.001$ \\
\hline
\end{tabular}

performed by a neuroradiologist (20 years of experience). Divergence between clinical and radiographic assessment was resolved by stereotactic biopsy.

\section{Data Processing and Quantitative Analysis}

All imaging data were analyzed using commercial software for MR imaging data (syngo.via; Siemens). An ROI was drawn to cover only the area of enhancement inside the lesions on postcontrast T1-weighted images (in which the target lesions have the largest extent of enhancement). Then, these ROIs were separately copied into T1-mapping images acquired at 5 and 60 minutes after contrast administration. Thus, $\mathrm{T} 1$ values at 5 minutes $\left(\mathrm{T} 1_{5 \min }\right)$ and 60 minutes $\left(\mathrm{T} 1_{60 \mathrm{~min}}\right)$ after contrast administration were acquired. Measurements of ROIs were performed 2 times for each patient's T1-mapping images, with an interval of 2 months between measurements. The average $\mathrm{T} 1$ value of each measurement was considered to represent the $\mathrm{T} 1$ value of the lesion. Then, the ms, we considered it qualified. Parameters for T1 mapping in the present study were as follows: FOV, $236 \times 236 \mathrm{~mm}$; slice thickness, $2.0 \mathrm{~mm}$; 80 axial slices; TE, $2.46 \mathrm{~ms}$; TR, $5.93 \mathrm{~ms}$; voxel size, $0.6 \times$ $0.6 \times 2.0 \mathrm{~mm}$; T1 estimate, $1000 \mathrm{~ms}$; the first flip angle, $2^{\circ}$; the second flip angle, $14^{\circ}$; bandwidth, $814 \mathrm{~Hz} / \mathrm{px}$; and generalized autocalibrating partially parallel acquisition reconstruction, 1 .

\section{DSC Perfusion Sequence}

The imaging parameters of DSC perfusion were as following: TR/ TE, 1600/30 ms; bandwidth, $1748 \mathrm{~Hz} /$ pixel; 21 axial slices; FOV, $220 \times 220 \mathrm{~mm}$; voxel size, $1.8 \times 1.8 \times 4 \mathrm{~mm}^{3}$; slice thickness, 4.0 $\mathrm{mm}$; and flip angle, $90^{\circ}$.

\section{Lesion Diagnosis}

Although histopathologic confirmation is the criterion standard for differentiating radionecrosis and tumor recurrence of BM after GKR, it could be obtained in only a small group of patients with BM in our study. Radiographic and clinical assessments were the most common methods to identify them in our center. Patients' conditions and lesions were thereafter regularly evaluated by clinical manifestations and routine MR imaging every 3 months. We made a final diagnosis of radiation necrosis when a target lesion showed complete response, partial response, or stable disease, depending on the Response Assessment in NeuroOncology-Brain Metastases method, on subsequent follow-up MR images for a minimum of 6 months. If the lesion presented with progression on serial MR imaging and the patient's neurologic condition deteriorated progressively, we diagnosed tumor recurrence. Clinical assessments were performed by a neurosurgeon (15 years of experience), and radiographic assessments were difference $\left(\mathrm{T} 1_{\text {differ }}\right)$ between these 2 values $\left(\mathrm{T} 1_{60 \text { min }}-\mathrm{T} 1_{5 \text { min }}\right)$ was calculated. To visually reflect the different components of mixed lesions, we created a $\mathrm{T} 1_{\text {differ }}$ map $\left(\mathrm{T} 1_{60 \mathrm{~min}}-\mathrm{T} 1_{5 \mathrm{~min}}\right)$. ROIs were also copied into the $\mathrm{CBV}$ map. The average value represents the value of enhancement of the lesion $\left(\mathrm{CBV}_{\text {lesion }}\right)$. Another ROI with a similar area was drawn on contralateral normal-appearing white matter. The average value represents the value of normalappearing white matter $\left(\mathrm{CBV}_{\mathrm{NAWM}}\right)$ : Mean $\mathrm{rCBV}=\mathrm{CBV}_{\text {lesion }}$ / $\mathrm{CBV}_{\text {NAWM}}$.

\section{Statistical Analysis}

Descriptive statistics (median and other measures) were determined for each parameter. Intergroup differences were tested for significance using the 2-tailed Mann-Whitney $U$ test. The Wilcoxon paired test was used to determine the significance of differences between $\mathrm{T} 1_{5 \mathrm{~min}}$ and $\mathrm{T} 1_{60 \mathrm{~min}}$ in both groups. For statistical analysis of T1-value trends, a trend map was calculated to demonstrate the trends across time for the 2 different processes. Correlation between $\mathrm{T} 1_{\text {differ }}$ and $\mathrm{rCBV}$ was tested by the Spearman correlation analysis. Sensitivity was defined as the ratio of accurately diagnosed recurrent metastases to the total number of recurrent metastatic lesions, and specificity was defined as the ratio of accurately diagnosed radionecrosis to the total number of radionecrotic lesions. Receiver operating characteristic curve analysis was used to determine the optimum cutoff values for differential diagnosis of recurrence and radionecrosis. $P<.05$ was considered statistically significant. All calculations were performed using SPSS (Version 20; IBM, Armonk, New York). 


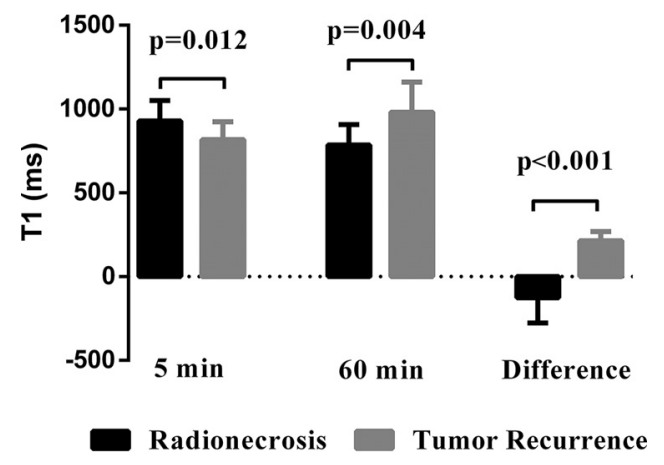

FIG 1. Comparison of $\mathrm{Tl}_{5 \mathrm{~min}}, \mathrm{Tl}_{60 \mathrm{~min}}$, and $\mathrm{Tl}_{\text {differ }}$ between the radionecrosis and tumor recurrence groups. There were significant differences in each of the 3 parameters $(P=.012, P=.004$, and $P<.001$, respectively) between the 2 groups.

\section{Correlation of $\mathrm{T}_{\text {differ }}$ and $\mathrm{rCBV}$}

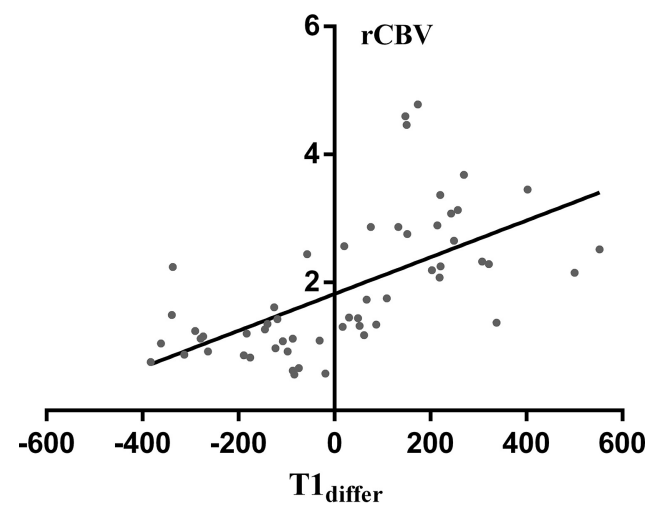

FIG 2. Correlation between $\mathrm{Tl}_{\text {differ }}$ and $\mathrm{rCBV}$. $\mathrm{Tl}_{\text {differ }}$ significantly correlated with $\mathrm{rCBV}(r=0.70 ; 95 \% \mathrm{Cl}, 0.53-0.82 ; P<.001)$.
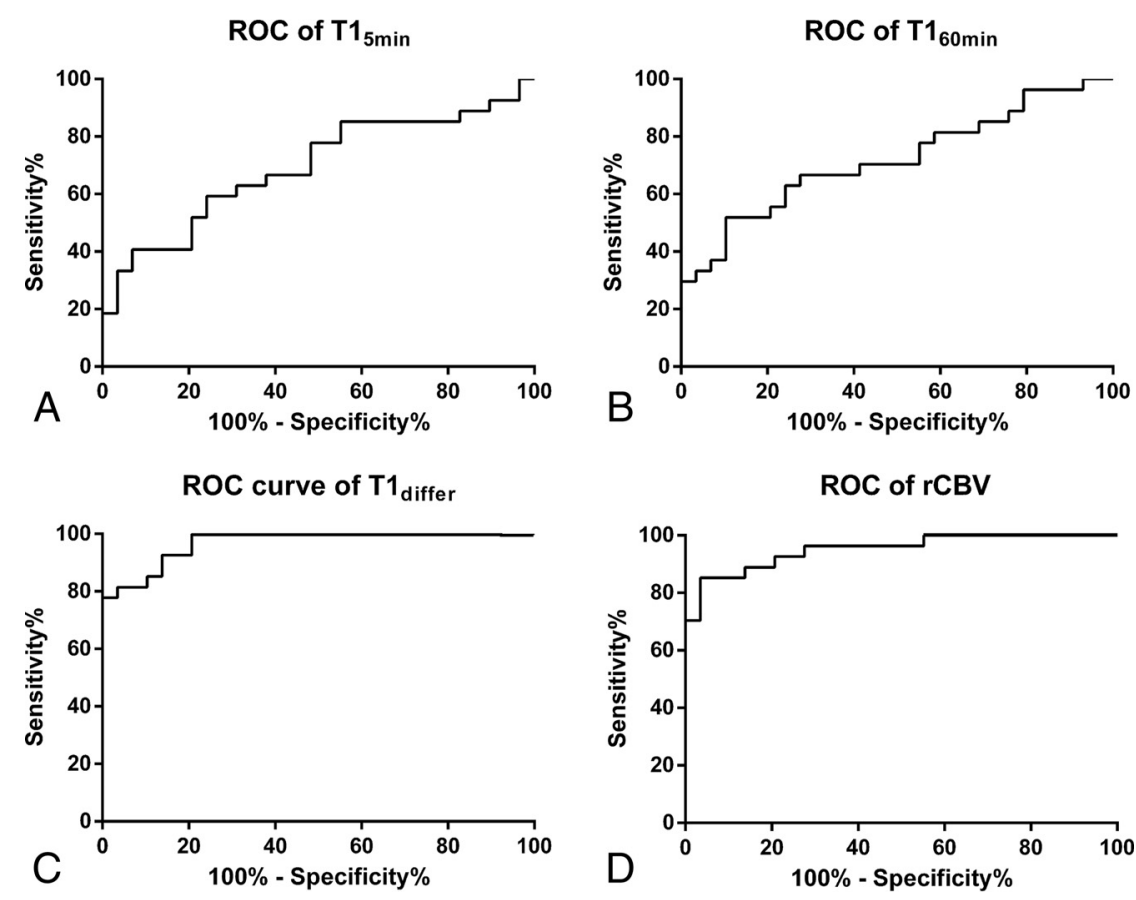

FIG 3. Receiver operating characteristic curves of $\mathrm{Tl}_{5 \min }(A), \mathrm{Tl}_{60 \mathrm{~min}}(B), \mathrm{Tl}_{\text {differ }}(C)$, and $\mathrm{rCBV}(D)$ for radionecrosis after stereotactic radiosurgery reveals that $\mathrm{Tl}_{\text {differ }}$ has a similar diagnostic performance compared with $\mathrm{rCBV}(\mathrm{AUC}=0.97 ; 95 \% \mathrm{Cl}, 0.93-1.00$ versus $\mathrm{AUC}=0.95 ; 95 \% \mathrm{Cl}, 0.90-1.00)$.

\section{RESULTS \\ Diagnostic Outcomes}

The clinical characteristics of the study population are given in Table 1 . None of the parameters were significantly different between the 2 groups. Eight (14.3\%) patients were diagnosed with tumor recurrence, and 5 patients $(8.9 \%)$ were diagnosed with radionecrosis based on histopathologic findings. Among the remaining lesions, 19 (33.9\%) and 24 (42.9\%), respectively, met the diagnostic criteria for tumor recurrence and radionecrosis. In total, $27(48.2 \%)$ and $29(51.8 \%)$ lesions were assigned to the tumor recurrence and radionecrosis groups, respectively.

\section{Quantitative Assessment}

Descriptive statistics for $\mathrm{T} 1_{5 \mathrm{~min}}, \mathrm{~T} 1_{60 \mathrm{~min}}, \mathrm{~T} 1_{\text {differ }}$, and $\mathrm{rCBV}$ of both groups are summarized in Table 2 . All the parameters differed significantly between the 2 groups $(P=.012, P=.004, P<$ .001 , and $P<.001$, respectively; Fig 1). Significant differences were observed between $\mathrm{T} 1_{60 \mathrm{~min}}$ and $\mathrm{T} 1_{5 \min }$ in both groups $(P<$ .001 , respectively). The radionecrosis group generally showed a decreasing trend in $\mathrm{T} 1$ values with time, while the recurrence group generally showed an increasing trend. $\mathrm{T} 1_{\mathrm{differ}}$ significantly correlated with the rCBV value $(r=0.70$; 95\% CI, 0.53-0.82; $P<$ .001 , Fig 2).The detailed information of each patient is shown in On-line Tables 1 and 2.

\section{Diagnostic Performance}

All parameters of the T1 map had a potential to distinguish radionecrosis from tumor recurrence. The receiver operating characteristic curve analysis indicated that $\mathrm{T} 1_{\text {differ }}$ (area under the curve [AUC] $=0.97 ; 95 \%$ CI, 0.93-1.00) had a higher diagnostic performance for discriminating radionecrosis and tumor recurrence than $\mathrm{T} 1_{5 \min }(\mathrm{AUC}=0.69 ; 95 \% \mathrm{CI}$, $0.55-0.83)$ and $\mathrm{T} 1_{60 \mathrm{~min}}(\mathrm{AUC}=0.72$; 95\% CI, 0.58-0.86; $P<.001$, respectively). However, $\mathrm{T} 1_{\text {differ }}$ did not perform significantly better than $\mathrm{rCBV}$ (AUC $=0.95 ; 95 \%$ CI, 0.90-1.00; $P=$ .274 , Fig 3$)$. The optimum $\mathrm{T} 1_{\text {differ }}$ for the differential diagnosis was $71.1 \mathrm{~ms}$, yielding an accuracy profile of the best sensitivity and specificity of $81.5 \%$ and $96.5 \%$, respectively (likelihood ratio $=$ 23.6). The optimum rCBV for the differential diagnosis was 1.74 , yielding an accuracy profile of the best sensitivity and specificity of $85.1 \%$ and $96.5 \%$, respectively (likelihood ratio $=24.7$ ). The optimum $\mathrm{T} 1_{5 \min }$ and $\mathrm{T} 1_{60 \mathrm{~min}}$ for differential diagnosis were 673.6 and $1086.0 \mathrm{~ms}$, respectively.

\section{DISCUSSION}

A high proportion of patients with $\mathrm{BM}$ have undergone GKR in the past 2 decades. ${ }^{26}$ Assessment of enhanced lesions after GKR, which constitutes a diagnostic dilemma, is an urgent requirement in 


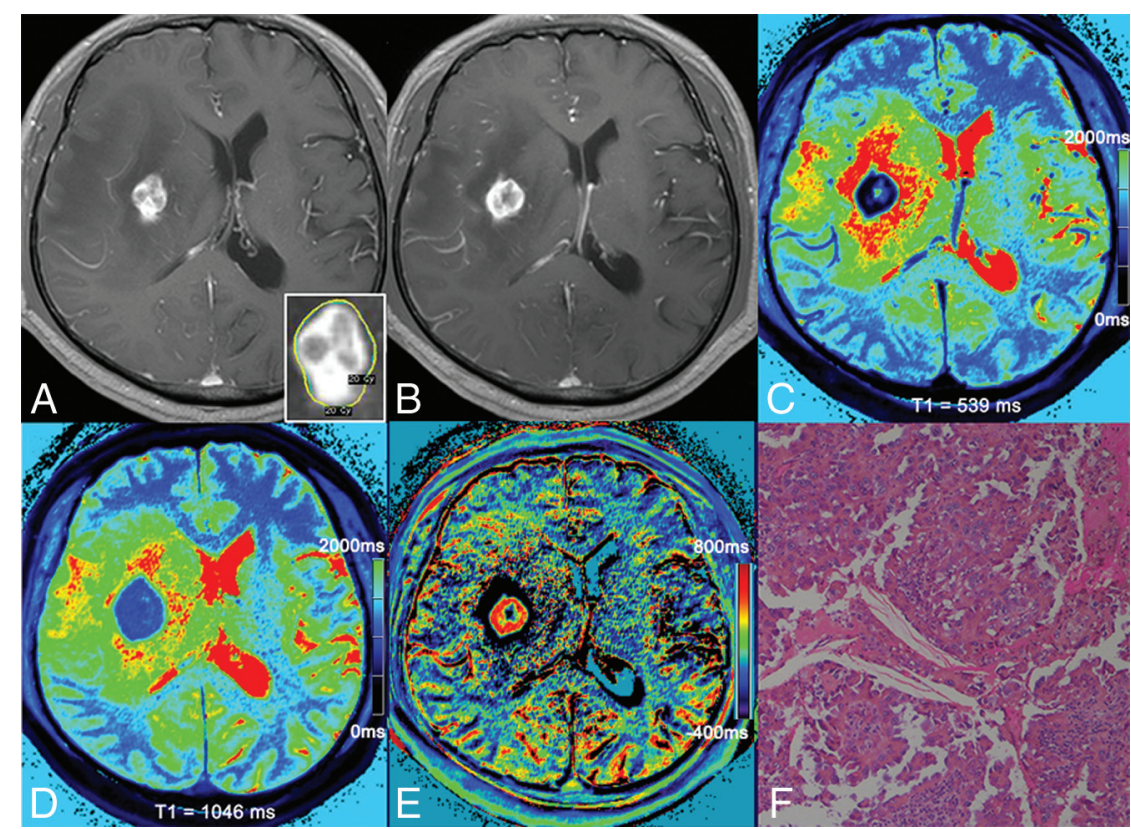

FIG 4. A 48-year-old male patient with cerebral metastasis from the lungs was treated with GKR (A). Follow-up MR imaging shows that enhancement returned at 5 months after GKR $(B)$, while T1 mapping 5 minutes $(C)$ and 60 minutes $(D)$ after contrast administration reveals $\mathrm{Tl}_{5 \mathrm{~min}}$ and $\mathrm{Tl}_{60 \mathrm{~min}}$ values of $539 \mathrm{~ms}$ and $1064 \mathrm{~ms}$, respectively, in the area of enhancement. The $T_{\text {differ }}$ map revealed a positive area in the lesion $(E)$. Histopathologic finings reveal lung cancer cells in the lesion $(F)$.

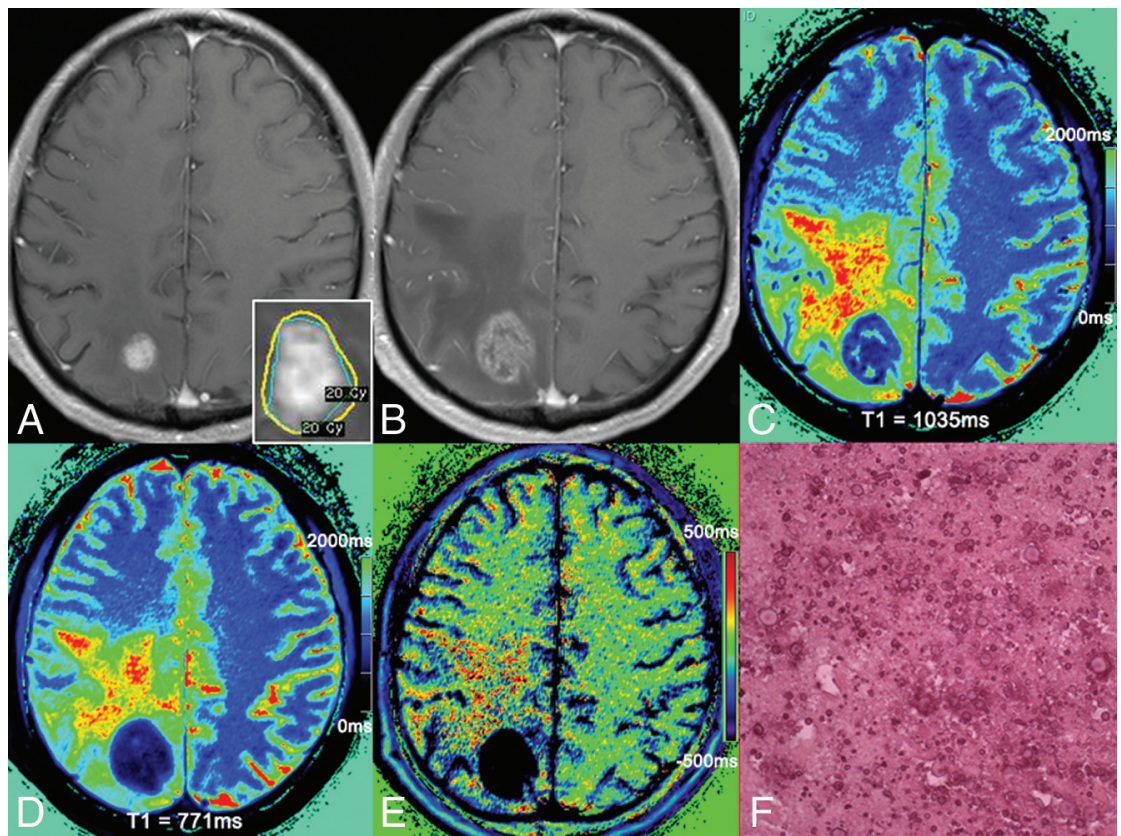

FIG 5. A 62-year-old female patient with brain metastasis from the breast was treated by GKR $(A)$. Follow-up MR imaging shows a $210 \%$ increment in maximal diameter at 6 months after GKR $(B)$. The results of 71 mapping at 5 minutes $(C)$ and 60 minutes $(D)$ after contrast administration reveal $\mathrm{Tl}_{5 \min }$ and $\mathrm{Tl}_{60 \mathrm{~min}}$ values of 1035 and $771 \mathrm{~ms}$, respectively, in the area of enhancement. The $\mathrm{Tl}_{\text {differ }}$ map reveals negative areas in the lesion (E). Histopathologic findings confirmed them as radionecrosis $(F)$.

the management of BM. ${ }^{4-6,27}$ In this study, with the help of a postcontrast T1 mapping technique, we present a new, high-resolution, quantitative method for resolving this issue.

Hemodynamic alteration in tumor recurrence is a consequence of a combination of intra- and extravascular aspects, including neoangiogenesis, vascular dilation, and the high permeability of morbid vessels inside tumors. ${ }^{28,29}$ The common feature of vessel morphology in the tumor area is undamaged vessel lumens. ${ }^{21}$ These combined pathophysiologic changes provide a basis for rapid contrast agent accumulation in the early period of contrast enhancement, resulting in an obvious decrease in $\mathrm{T} 1$ values. On the other hand, the compact structure of tumors prevents continuous accumulation of contrast agent, and more important, the undamaged vessel lumens contribute to the fast clearance of contrast agent in the late period, ${ }^{30,31}$ resulting in an increase in $\mathrm{T} 1$ values compared with those in the early period. Therefore, the positive part within the irradiated area in the $\mathrm{T} 1_{\text {differ }}$ map represents the tumor recurrence. Figure 4 shows an example of T1 mapping of tumor recurrence.

Although enhancement of radionecroses also results from increased vascular permeability, there is an obvious difference in the hemodynamic alteration between radionecrosis and tumor recurrence. In contrast to the rapid contrast agent accumulation in tumor recurrence, transport of contrast agent in radionecrosis is a consequence of slow permeability. ${ }^{32,33}$ In radionecrosis, vessels present with significantly damaged lumens. ${ }^{21}$ In addition, large extravascular and extracellular spaces can be found within the entity. ${ }^{22,23}$ These changes contribute to the continuous-but-slow accumulation of contrast agent. ${ }^{19,22}$ In addition, the damaged vessel lumens and lack of neovascularization prevent rapid clearance of the contrast agent in the late period, resulting in a decrease in $\mathrm{T} 1$ values relative to those in the early period. Therefore, the negative part within the irradiated area in the $\mathrm{T} 1_{\text {differ }}$ map represents radionecrosis. Figure 5 shows an example of $\mathrm{T} 1$ mapping in radionecrosis.

Previous studies have focused on measuring changes in signal intensity using contrast-enhanced T1WI sequences. $^{27,34,35}$ Recently, Wagner et $\mathrm{al}^{27}$ found that subtraction of signal intensity is efficient in the differential diagnosis between radionecrosis and tumor recurrence. In comparison with signal intensity, T1 mapping has the following advantages: First, the T1 value is a quantitative metric, which enables the intra- and interpatient comparison across different institutions. Second, quantitative $\mathrm{T} 1_{\text {differ }}$ mapping could better reflect the heterogeneity of enhanc-

AJNR Am J Neuroradiol 39:1025-31 Jun 2018 www.ajnr.org 


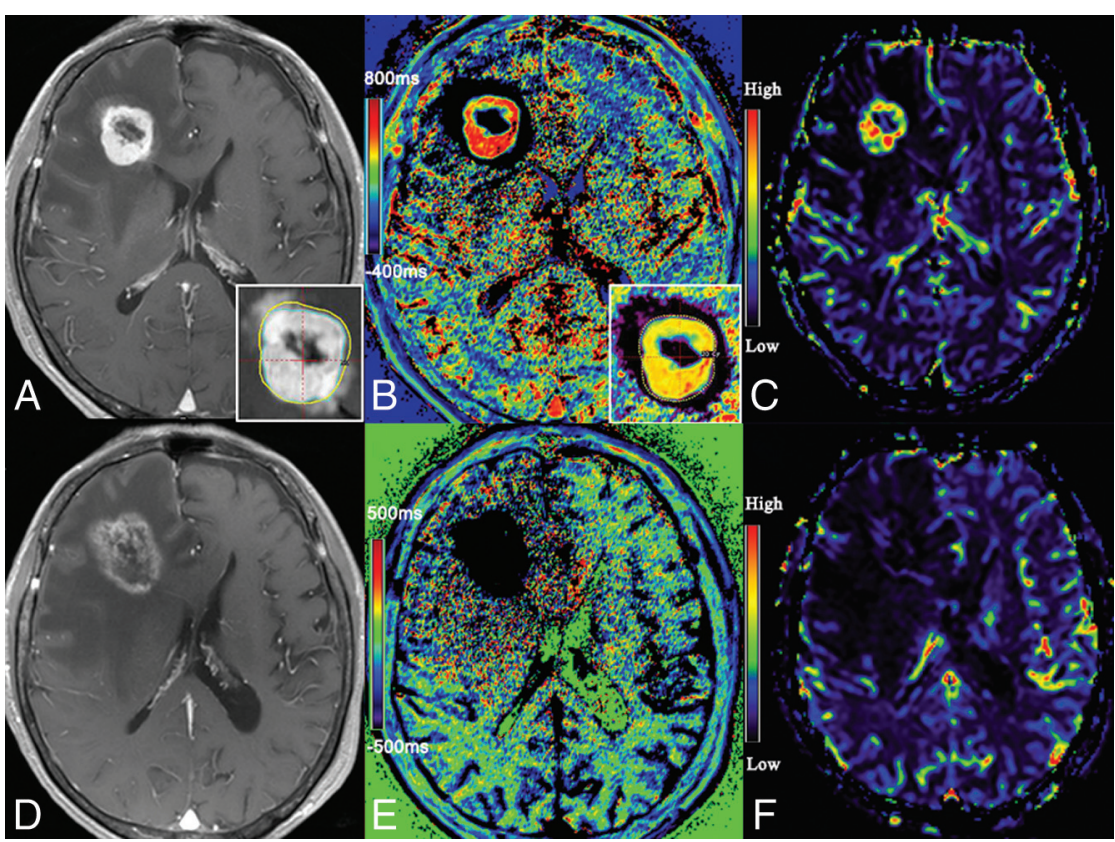

FIG 6. MR image of a 54-year-old female patient with cerebral metastasis from the digestive tract. Contrast-enhanced T1-weighted image shows enhancement in the previously irradiated lesion 6 months later, after the first GKR $(A)$. The $T_{\text {differ }}$ map demonstrates it as a mixed lesion and detects the parts of tumor recurrence to guide the treatment plan $(B)$. The recurrent part is confirmed by a cerebral blood volume map (C). Follow-up MR imaging (6 months after re-irradiation) shows a $90 \%$ increment in maximal diameter $(D)$. The $T 1_{\text {differ }}$ map demonstrates it as radionecrosis $(E)$. The radionecrosis is confirmed by a CBV map $(F)$.

ing lesions (subvolume of lesions) in an objective approach. These advantages suggest that $\mathrm{T} 1$ mapping may be more practical in clinical diagnosis and treatment. However, whether subtraction of T1 mapping has a higher resolution than signal intensity remains uncertain. Furthermore, we made progress in the methodology, in which we used a gamma knife rigid head frame and Brain Dot Engine technique to guarantee identical slice position and angulation. Thus, the subtraction of T1 mapping could be precisely done at the voxel-to-voxel level.

Although the present results showed that $\mathrm{T} 1_{5 \mathrm{~min}}, \mathrm{~T} 1_{60 \mathrm{~min}}$, and $\mathrm{T} 1_{\text {differ }}$ could help distinguish radionecrosis and tumor recurrence, we regard $\mathrm{T} 1_{\text {differ }}$ as a better parameter for the differential diagnosis. Theoretically, $\mathrm{T} 1_{\text {differ }}$ should be negative in radionecrosis and positive in recurrence. In the present study, 5 patients with radionecrosis and 2 with recurrence showed opposite tendencies. Nevertheless, the $\mathrm{T} 1_{\text {differ }}$ values were all within a reliable interval. The presence of an admixture of components in lesions might be responsible for this discrepancy. Thus, we conclude that the ratio of tumor recurrence to radionecrosis is the key point underlying this bias because these 2 opposite hemodynamic compartments may counteract each other to some extent. This is also an inherent pitfall accompanying other techniques for identifying tumor recurrence and radionecrosis.

Our results demonstrate that $\mathrm{T}_{\text {differ }}$ correlated with $\mathrm{rCBV}$, and they had similar diagnostic performances. Even so, in comparison with DSC perfusion, T1 mapping has many advantages: First, T1 mapping is a quantitative imaging method. Second, quantitative $\mathrm{T} 1_{\text {differ }}$ mapping could better reflect the heterogeneity in enhancing lesions. Third, T1 mapping is less sensitive to susceptibility artifacts. Fourth, bolus injection with a high velocity is not necessary for T1 mapping. Thus, patients who are taking chemotherapeutic drugs may benefit because their vessels are too fragile to accept this velocity. Last, T1 mapping has a better coregistration with anatomic images, a great advantage in that $\mathrm{T} 1_{\text {differ }}$ could be used for the re-irradiation of recurrent $\mathrm{BM}$ in the gamma knife planning system. Figure 6 shows an example of a $\mathrm{T} 1_{\text {differ }}$ map guiding the precise treatment of recurrent tumor using a radiation-treatment-planning system. On the basis of these advantages, we strongly recommend $\mathrm{T} 1$ mapping being performed in the patients who could benefit from this technique.

Some limitations of this study should be addressed here. First, only 13 patients were diagnosed by histopathologic examination after GKR. For patients with a high probability of radionecrosis, this is an inherent dilemma in clinical treatment because the risk of possible complications of biopsy might outweigh the benefits of the histopathologic diagnosis. In addition, the long observation period justifies the diagnosis of the remaining lesions based on MR imaging data. Second, a limited number of patients were recruited in this study. However, the present study group was nearly homogeneous, and all data were acquired through a consistent MR imaging protocol.

\section{CONCLUSIONS}

Taking advantage of the postcontrast T1 mapping technique, we set up a new method for assessing the treatment response after GKR for BM. Our results show that radionecrosis and tumor recurrence exhibit different trends for T1 values after Gd-DTPA administration. Although $\mathrm{Tl}_{5 \mathrm{~min}}, \mathrm{Tl}_{60 \mathrm{~min}}$, and $\mathrm{T} 1_{\text {differ }}$ differ significantly between the 2 groups in the present study, $\mathrm{Tl}_{\text {differ }}$ is a better differential marker for radionecrosis and tumor recurrence. Despite some limitations of this study, we believe that the benefits of T1 mapping will promote its wide application and provide more concrete evidence for treatment of $\mathrm{BM}$ in the future.

Disclosures: Yingchao Liu—RELATED: Grant: National Natural Science Foundation of China (81641176), Natural Science Foundation of Shandong Province (ZR20141TM002), Science and Technology Planning Project of Shandong Province (2014GSFl18046, 2015GSF18161, 2016GSF201092, and 2017CXGC1209), and Taishan Scholars Program (No. tsqn20161070). Shangchen Xu—RELATED: Grant: Natural Science Foundation of Shandong Province (ZR2014HM069).

\section{REFERENCES}

1. Patel TR, Knisely JP, Chiang VL. Management of brain metastases: surgery, radiation, or both? Hematol Oncol Clin North Am 2012;26: 933-47 CrossRef Medline

2. Pinkham MB, Whitfield GA, Brada M. New developments in intra- 
cranial stereotactic radiotherapy for metastases. Clin Oncol ( $R$ Coll Radiol) 2015;27:316-23 CrossRef Medline

3. Mahajan A, Ahmed S, McAleer MF, et al. Post-operative stereotactic radiosurgery versus observation for completely resected brain metastases: a single-centre, randomised, controlled, phase 3 trial. Lancet Oncol 2017;18:1040-48 CrossRef Medline

4. Curnes JT, Laster DW, Ball MR, et al. MRI of radiation injury to the brain. AJR Am J Roentgenol 1986;147:119-24 CrossRef Medline

5. Lyubimova N, Hopewell JW. Experimental evidence to support the hypothesis that damage to vascular endothelium plays the primary role in the development of late radiation-induced CNS injury. $\mathrm{Br} \mathrm{J}$ Radiol 2004;77:488-92 CrossRef Medline

6. Stockham AL, Tievsky AL, Koyfman SA, et al. Conventional MRI does not reliably distinguish radiation necrosis from tumor recurrence after stereotactic radiosurgery. J Neurooncol 2012;109:149-58 CrossRef Medline

7. Brandsma D, Stalpers L, Taal W, et al. Clinical features, mechanisms, and management of pseudoprogression in malignant gliomas. Lancet Oncol 2008;9:453-61 CrossRef Medline

8. Verma N, Cowperthwaite MC, Burnett MG, et al. Differentiating tumor recurrence from treatment necrosis: a review of neuro-oncologic imaging strategies. Neuro Oncol 2013;15:515-34 CrossRef Medline

9. Dhermain FG, Hau P, Lanfermann H, et al. Advanced MRI and PET imaging for assessment of treatment response in patients with gliomas. Lancet Neurol 2010;9:906-20 CrossRef Medline

10. Barajas RF, Chang JS, Sneed PK, et al. Distinguishing recurrent intra-axial metastatic tumor from radiation necrosis following gamma knife radiosurgery using dynamic susceptibility-weighted contrast-enhanced perfusion MR imaging. AJNR Am J Neuroradiol 2009;30:367-72 Medline

11. Mitsuya K, Nakasu Y, Horiguchi S, et al. Perfusion weighted magnetic resonance imaging to distinguish the recurrence of metastatic brain tumors from radiation necrosis after stereotactic radiosurgery. J Neurooncol 2010;99:81-88 CrossRef Medline

12. Mills SJ, Soh C, O'Connor JP, et al. Enhancing fraction in glioma and its relationship to the tumoral vascular microenvironment: a dynamic contrast-enhanced MR imaging study. AJNR Am J Neuroradiol 2010;31:726-31 CrossRef Medline

13. Larsen VA, Simonsen HJ, Law I, et al. Evaluation of dynamic contrast-enhanced T1-weighted perfusion MRI in the differentiation of tumor recurrence from radiation necrosis. Neuroradiology 2013; 55:361-69 CrossRef Medline

14. Koh MJ, Kim HS, Choi CG, et al. Which is the best advanced MR imaging protocol for predicting recurrent metastatic brain tumor following gamma-knife radiosurgery: focused on perfusion method. Neuroradiology 2015;57:367-76 CrossRef Medline

15. Fugo KR, Lawson MA, Lee BJ. Ibotenic acid lesions reduce noradrenergic activation in ventromedial hypothalamus during hypoglycemia. Brain Res 2006;1111:105-10 CrossRef Medline

16. Biousse V, Newman NJ, Hunter SB, et al. Diffusion weighted imaging in radiation necrosis. J Neurol Neurosurg Psychiatry 2003;74: 382-84 CrossRef Medline

17. Weybright P, Sundgren PC, Maly P, et al. Differentiation between brain tumor recurrence and radiation injury using MR spectroscopy. AJR Am J Roentgenol 2005;185:1471-76 CrossRef Medline

18. Smith EA, Carlos RC, Junck LR, et al. Developing a clinical decision model: MR spectroscopy to differentiate between recurrent tumor and radiation change in patients with new contrast-enhancing lesions. AJR Am J Roentgenol 2009;192:W45-52 CrossRef Medline
19. Mahrholdt H. Delayed enhancement cardiovascular magnetic resonance assessment of non-ischaemic cardiomyopathies. Eur Heart J 2005;26:1461-74 CrossRef Medline

20. Zach L, Guez D, Last D, et al. Delayed contrast extravasation MRI: a new paradigm in neuro-oncology. Neuro Oncol 2015;17:457-65 CrossRef Medline

21. Zach L, Guez D, Last D, et al. Delayed contrast extravasation MRI for depicting tumor and non-tumoral tissues in primary and metastatic brain tumors. PLoS One 2012;7:e52008 CrossRef Medline

22. Wong CS, Van der Kogel AJ. Mechanisms of radiation injury to the central nervous system: implications for neuroprotection. Mol Interv 2004;4:273-84 CrossRef Medline

23. Chamberlain MC, Glantz MJ, Chalmers L, et al. Early necrosis following concurrent Temodar and radiotherapy in patients with glioblastoma. J Neurooncol 2007;82:81-83 CrossRef Medline

24. Wang HZ, Riederer SJ, Lee JN. Optimizing the precision in T1 relaxation estimation using limited flip angles. Magn Reson Med 1987;5: 399-416 CrossRef Medline

25. Yarnykh VL. Actual flip-angle imaging in the pulsed steady state: a method for rapid three-dimensional mapping of the transmitted radiofrequency field. Magn Reson Med 2007;57:192-200 CrossRef Medline

26. Andrews DW, Scott CB, Sperduto PW, et al. Whole brain radiation therapy with or without stereotactic radiosurgery boost for patients with one to three brain metastases: phase III results of the RTOG 9508 randomised trial. Lancet 2004;363:1665-72 CrossRef Medline

27. Wagner S, Gufler H, Eichner G, et al. Characterisation of lesions after stereotactic radiosurgery for brain metastases: impact of delayed contrast magnetic resonance imaging. Clin Oncol ( $R$ Coll Radiol) 2017;29:143-50 CrossRef Medline

28. Aronen HJ, Gazit IE, Louis DN, et al. Cerebral blood volume maps of gliomas: comparison with tumor grade and histologic findings. $R a-$ diology 1994;191:41-51 CrossRef Medline

29. Cha S, Johnson G, Wadghiri YZ, et al. Dynamic, contrast-enhanced perfusion MRI in mouse gliomas: correlation with histopathology. Magn Reson Med 2003;49:848-55 CrossRef Medline

30. Hompland T, Gulliksrud K, Ellingsen C, et al. Assessment of the interstitial fluid pressure of tumors by dynamic contrast-enhanced magnetic resonance imaging with contrast agents of different molecular weights. Acta Oncol 2013;52:627-35 CrossRef Medline

31. Watling CJ, Lee DH, Macdonald DR, et al. Corticosteroid-induced magnetic resonance imaging changes in patients with recurrent malignant glioma. J Clin Oncol 1994;12:1886-89 CrossRef Medline

32. Lüdeman L, Grieger W, Wurm R, et al. Glioma assessment using quantitative blood volume maps generated by T1-weighted dynamic contrast-enhanced magnetic resonance imaging: a receiver operating characteristic study. Acta Radiol 2006;47:303-10 CrossRef Medline

33. Lüdeman L, Grieger W, Wurm R, et al. Quantitative measurement of leakage volume and permeability in gliomas, meningiomas and brain metastases with dynamic contrast-enhanced MRI. Magn Reson Imaging 2005;23:833-41 CrossRef Medline

34. Mathews VP, Caldemeyer KS, Ulmer JL, et al. Effects of contrast dose, delayed imaging, and magnetization transfer saturation on gadolinium-enhanced MR imaging of brain lesions. J Magn Reson Imaging 1997;7:14-22 CrossRef Medline

35. Schörner W, Laniado M, Niendorf HP, et al. Time-dependent changes in image contrast in brain tumors after gadolinium-DTPA. AJNR Am J Neuroradiol 1986;7:1013-20 Medline 